\title{
Metodologias Ativas no Ensino de Teste de Software para Alunos com Dedicação Parcial
}

\author{
Andressa de Souza Silva Medeiros \\ Universidade Estadual de Mato Grosso do Sul \\ Nova Andradina, MS, Brasil \\ andressasilva.0797@gmail.com
}

\author{
Jorge Marques Prates \\ Universidade Estadual de Mato Grosso do Sul \\ Dourados, MS, Brasil \\ jprates@uems.br
}

\begin{abstract}
The relationship between the university and its audience has been changed, the place that only used to graduate students of high social standard, nowadays it graduate students of all social classes. As a result, there are students who can not only play the role of student, some have to work to maintain themselves, becoming a student with partial dedication. These students, in most cases, end up not enjoying all the possibilities that the university offers and may be impaired by losing experiences that would assist you in your academic life. In this context, the purpose of this work was to use the active methodologies: problem-based and collaborative learning in Software Engineering teaching to help students who are partially dedicated, adapting alternative ways to fix knowledge. For the application of the proposal, some students were invited, and divided into two classes, in which one of them used the traditional method and the other applied active methodologies were used. In addition, the class that used the active methodologies, had the help of app created so that they could prepare for the class. To obtain the results, forms were available to verify the students' opinion regarding the applied method. These were satisfactory, since according to the them, the problem-based and collaborative learning proved to be beneficial, the app also brought advantages such as its practicality, easy-to-understand content, and the possibility of using in free time.
\end{abstract}

\section{KEYWORDS}

Ensino de engenharia de software, teste de software, aprendizagem baseada em problemas, aprendizagem colaborativa, alunos com dedicação parcial

\section{INTRODUÇÃO}

Uma educação de qualidade resulta em instruir informações aos educandos de maneira que possam adquirir base para a construção de seu conhecimento [1]. Quando as práticas pedagógicas tradicionais são analisadas, é visto que as mesmas constituem apenas em aulas expositivas em que o aluno apenas lê, escreve e memoriza informações passadas por seus professores. Entretanto, ensinar não é somente transferir conhecimento.

No âmbito dos estudos, alguns alunos não conseguem se dedicar totalmente aos estudos caracterizando-os como alunos com dedicação parcial. A partir do momento em que o estudante tem que dividir seu tempo entre trabalho e estudo, o segundo acaba ficando parcialmente afetado. Dessa maneira, o trabalho dificulta o envolvimento do aluno em seu meio acadêmico, tornando o aprendizado um tanto quanto mais complexo [2].

\begin{abstract}
A Engenharia de Software é uma disciplina presente em todos os cursos de graduação que tem a Computação como atividade meio ou atividade fim. A mesma é responsável por englobar todos os assuntos relacionados a um sistema, desde o que será feito, até sua manutenção, visando garantir padrões de qualidade ao software desenvolvido.

O objetivo geral deste trabalho foi investigar e aplicar as metodologias ativas, especificamente a aprendizagem baseada em problemas e a colaborativa, no estudo de Engenharia de Software. Como estudo de caso foram desenvolvidas atividades no contexto de teste de software. Com isso, foi possível investigar como amenizar os fatores dificultadores da aprendizagem dos alunos com dedicação parcial nesta disciplina. Para atingir o objetivo geral, inicialmente foram identificados fatores que dificultam o aprendizado, de um modo geral, dos alunos que se dedicam parcialmente. Além disso, para apoiar as metodologias ativas, foi desenvolvida uma ferramenta a fim de auxiliar na fixação do conteúdo de teste de software.

Na Seção 2 apresenta-se a contextualização das metodologias ativas com ênfase na aprendizagem colaborativa e aprendizagem baseada em problemas. Já a apresentação dos conceitos, quem são e as características referentes aos alunos com dedicação parcial são apresentados na Seção 4.

A proposta deste artigo é exposta na Seção 5. Já a Seção 6 apresenta a discussão dos resultados obtidos a partir da aplicação da proposta. Para finalizar, a Seção 7 apresenta as considerações finais referentes aos resultados alcançados.
\end{abstract}

\section{METODOLOGIAS ATIVAS}

As metodologias ativas atuam no ensino centrado no aluno, ou seja, o aprendizado baseia-se no aluno como centro do processo de ensino. Logo, no método ativo as aulas passam de meramente expositivas para aulas extremamente interativas, focando na prática e envolvendo o aluno. Essas metodologias fundamentam-se no desenvolvimento do processo de aprender, fazendo uso de experiências reais ou simuladas, com a intensão de solucionar desafios oriundos de atividades essenciais em diferentes contextos [3].

$\mathrm{Na}$ atualidade é muito importante o uso de metodologias ativas para o ensino, pois com o avanço das tecnologias é possível que nos próximos anos o ensino tradicional tenha se transformado. A modernização vem trazendo o progresso dessas tecnologias, as quais, quando adequadas, podem ser grandes aliadas aos métodos ativos da aprendizagem [4].

Dentre as abordagens ativas estão a aprendizagem baseada em problemas (ABP) (do inglês problem-based learning - $\mathrm{PBL}$ ), aprendizagem baseada em projetos, aprendizagem em pares, aprendizagem colaborativa, aprendizagem baseada em jogos, gamificação. 
Nesse trabalho foram utilizadas as abordagens da aprendizagem colaborativa e a aprendizagem baseada em problemas.

\subsection{Aprendizagem Colaborativa}

A aprendizagem colaborativa é a prática que instiga o indivíduo a interagir e produzir em grupo, além de adquirir competências para pesquisar a fim de construir seu próprio conhecimento. Sendo estes alguns dos requisitos mais vislumbrados e valorizados pelas empresas [5].

A aprendizagem colaborativa é uma das abordagens de ensino que tem chamado a atenção de pesquisadores da área da educação. Uma vez que, além de ser uma metodologia de ensino que envolve a colaboração dos educandos no processo de ensino-aprendizagem, ainda é uma metodologia multidisciplinar, ou seja, que pode ser aplicada em diversas disciplinas.

Embora o conceito de aprendizagem colaborativa pareça novo, ele tem sido testado e aperfeiçoado por pesquisadores desde o século XVIII. Com a evolução das tecnologias, atualmente, é imprescindível deixá-las de lado em relação a temática educação, tendo em vista que as tecnologias podem ser um fator de motivação para os educandos. Quando são utilizadas práticas pedagógicas inovadoras é produzido um novo cenário que pode gerar entusiasmo ao aluno, levando-o a produção do conhecimento [6].

$\mathrm{Na}$ aprendizagem colaborativa os estudantes desenvolvem algumas características. O raciocínio, respeito com a opinião do outro, e o poder de persuasão são exemplos delas. Além disso, concentra-se no aluno, colaborando para a produção do conhecimento. Durante as discussões, as explicações de um estudante podem ser mais compreensíveis do que as do professor. Isso se deve ao fato de que o estudante acabou de adquirir o conhecimento e sabe quais foram as principais dificuldades e/ou dúvidas que apresentou durante o aprendizado [7].

Na metodologia colaborativa, à medida que o aluno está ativo no processo de construção do seu conhecimento ele tende a produzir encorajamento para pesquisar, dialogar, para ir em busca da sua aprendizagem [5]. No entanto, é importante que na aprendizagem colaborativa o aluno não utilize a pesquisa somente como forma de obtenção do conhecimento para análise teórica, mas que isso torne-se natural para a sua própria realidade de vida [8].

\subsection{Aprendizagem Baseada em Problemas (ABP)}

A ABP surgiu em meados do século de 1960 na Universidade de McMaster, no Canadá. A princípio esta metodologia foi utilizada em cursos de Medicina, a fim de superar a ausência das relações entre a teoria e a prática relacionando o teórico com o contexto profissional real [9].

A ABP é fundamentada em princípios que mostram que a aprendizagem não está relacionada ao processo de recepção passiva e acumulo de informações, mas na construção de conhecimentos [10]. O aprendizado por meio da problematização é uma possibilidade de tornar o aluno ativo para seu próprio processo de formação [11]. Portanto, a ABP é uma metodologia baseada na resolução de problemas. Todavia, essa abordagem não se baseia apenas na resolução de problemas contínuo, é exigido uma lógica para que seja executada com sucesso.
Na maioria das metodologias de ensino tradicionais, o professor (emissor) apresenta ao aluno (receptor) o conteúdo. Em seguida é realizada a explicação do conteúdo para depois diversos exemplos serem realizados. Após todos esses passos, são apresentados para os alunos exercícios referentes a este conteúdo. Já na ABP, uma de suas características que se destaca é o processo do aluno se depara com a situação problema precedendo o conceito para a sua solução, conduzindo o aluno a motivação inicial para a busca do conhecimento [12].

O propósito da ABP é instigar no aluno a prática do estudo e do pensamento reflexivo, além de promover a autonomia da aprendizagem, convívio e trabalho em equipe, já que é isto que se espera de um profissional em um ambiente real. Logo, é interessante que o problema apresentado ao aluno seja relevante ou muito próximo ao que ele irá se deparar no âmbito profissional [9].

Assim, o aluno aprende a pensar não só por aprender os princípios para resolver problemas, mas pelas séries de estratégias mentais que os levam a sua resolução. Já que para isso são necessárias a combinação de fontes conhecidas e a busca eminente pelo desconhecido [13].

Um conceito relacionado a $\mathrm{ABP}$ é a transferência do meio real para o meio teórico. Uma vez que, conforme [14], "[...] com problemas reais, o discente costuma estar muito mais motivado para examinar, refletir e pode relacionar sua história ao que é investigado, ressignificando suas descobertas". Ainda de acordo com [14], a utilização de problemas no ensino facilita o contato com as informações, possibilitando desta forma, os impasses para com o acesso ao aprendizado.

A ABP funciona com algumas práticas para que o seu ensino se torne de qualidade, até mesmo porque não basta ter uma metodologia inovadora sem regras que a torne realmente de qualidade. Logo, ela apresenta alguns papéis e etapas, os quais serão descritos a seguir.

Os papéis deste método podem se dividir em tutor, coordenador e secretário. $\mathrm{O}$ tutor tem a função desempenhada pelo professor, que neste contexto tem apenas o posto de mediador, ele deve auxiliar com sugestões, além de motivar os discentes a participarem e estimular o trabalho em grupo. Já o coordenador e o secretário, têm a função desempenhada por alunos, os quais um deve garantir que as etapas da $\mathrm{ABP}$ sejam cumpridas até o fim da tutoria e o outro registra as cláusulas do problema, respectivamente [15].

As funções desenvolvidas pelos alunos se alternam a cada problema, ou seja, a cada novo problema a ser discutido uma nova dupla de alunos se forma para que todos tenham um primeiro contato, desempenhem e desenvolvam a função da gerência [15].

Portanto, a ABP é uma metodologia que contribui no apoio ao trabalho em grupo promovendo a aprendizagem colaborativa, fazendo com que os discentes aprendam a respeitar as opiniões bem como a persuadir e ter poder de argumentação, além de auxiliar o discente a ter autonomia para estudos independentes, os quais são especificidades que as empresas buscam, atualmente, no mercado de trabalho.

\section{ENSINO DE ENGENHARIA DE SOFTWARE}

O ensino de Engenharia de Software está contido em quase todos os cursos que tem a Computação como atividade meio ou fim 
[16]. Visto que, a mesma é responsável por englobar todos os assuntos relacionados a um sistema, desde o que será feito até sua manutenção visando garantir padrões de qualidade ao software desenvolvido.

Segundo a Sociedade Brasileira de Computação - SBC, para que se possa obter softwares confiáveis e que funcionem com eficiência em máquinas reais, é necessário que seja estabelecido princípios sólidos de engenharia. Além disso, a Engenharia de Software concentra sua preocupação em controlar todas as fases do processo de desenvolvimento do software por meio de métricas para garantir o controle produtivo dessas aplicações. [16]

Sommerville [17], define a Engenharia de Software como "uma disciplina de engenharia que está focada em todos os aspectos da produção de um software, desde o princípio da extração dos requisitos até a manutenção do software que já está em uso".

Portanto, a Engenharia de Software se tornou e é fundamental para o currículo de um aluno da área da Computação. É importante salientar, que todo aluno dessa área deve conhecer e compreender os princípios básicos da Engenharia de Software, bem como aplicálas da maneira correta. Em consequência disto, é fundamental ter um bom aprendizado sobre a teoria e a prática da disciplina referida.

Todavia, como o ensino de Engenharia de Software requer um nível alto de absorção dos conceitos teóricos e práticos, na maioria das vezes, o ensino-aprendizado se torna um real desafio para quem leciona e para quem aprende [18]. Além disso, a carga horária da disciplina é muito curta para tratar todos os princípios da matéria, bem como para conseguir realizar aulas práticas de qualidade.

Para alcançar os objetivos da referida disciplina, podem ser utilizadas metodologias de ensino-aprendizado que optam em aderir às melhores práticas pedagógicas. Dentre as metodologias utilizadas a que mais se destaca é a do aprendizado centrado no aluno, a qual utiliza abordagens ativas e práticas para desenvolver no aluno o raciocínio para chegar na solução de problemas [16].

\subsection{Aprendizagem Baseada em Problemas no Ensino de Engenharia de Software}

A Engenharia de Software é uma disciplina importante nos cursos de Computação, visto que ela se encontra presente na maioria deles. Desta maneira, é necessário que seu ensino seja bem absorvido por estudantes desta área. Existem diversas pesquisas sobre a inserção de metodologias ativas no ensino de Engenharia de Software. Por exemplo, a metodologia baseada em projetos, com o objetivo de torná-la um pouco mais prática, fazendo com que esta seja mais atrativa para os estudantes.

Entretanto, os pequenos projetos desenvolvidos podem não trazer toda a experiencia necessária para quem vai trabalhar nesta área, haja visto que os mini projetos inseridos na disciplina são realizados muito rapidamente sem muito aprofundamento. Além disso, uma das desvantagens desta abordagem é o tempo longo para aplicação [19].

Uma metodologia ativa que vem sendo utilizada nesta disciplina é a aprendizagem baseada em problema (ABP). Esta abordagem, quando bem preparada e aplicada, traz uma gama de vantagens para os educandos, promovendo autonomia, raciocínio, trabalho em equipe, além de contar com problemas que se assemelham a uma situação real utilizando as competências exibidas em aula.
Já [20], afirmam que o conteúdo pode ser um pouco difícil para os alunos compreenderem, e quando é utilizada uma metodologia tradicional pode não possibilitar toda a experiência necessária para que o aluno o aplique em um cenário real. Segundo os autores, o problema é a motivação inicial para iniciar a aprendizagem, ou seja, o aprendizado centrado no aluno incentiva o educando a utilizar os saberes curriculares e extracurriculares, para além disso provocar o estimulo em busca de novos conhecimentos, os quais são necessários para resolução do problema.

Para [21], esporadicamente, existem reclamações de empresas de engenharia de software, em relação a competência prática de muitos profissionais que acabam de sair de uma universidade. Uma vez que, estes podem ter um elevado conhecimento teórico, porém muitas vezes não estão habituados com os problemas frequentes que encontram na realidade. Por este motivo, a pesquisa de [21] consistiu em uma abordagem que reunisse a aprendizagem baseada em problemas e a aprendizagem baseada em projetos para mostrar aos alunos os problemas que podem surgir ao desenvolverem projetos reais.

Conforme [22], existe uma certa dificuldade em encontrar profissionais capacitados na área da informática, como um engenheiro de software. Ainda citam que pode ser notado que os métodos tradicionais de aprendizagem podem ser um dos fatores que implicam essa falta de qualificação, já que com este método de ensino não existe uma contribuição significativa em relação ao desenvolvimento do educando.

Nesta perspectiva, a utilização de abordagens de ensino ativas como o método ABP pode ajudar a solucionar este obstáculo. No intuito de formar profissionais mais qualificados para o mercado de trabalho os autores [22] utilizaram a aprendizagem baseada em problemas juntamente com a fábrica de software, que visa trazer clientes reais com problemas reais para os alunos tornando-os mais experientes.

Quando o problema trabalhado se assemelha a uma prática profissional ou a uma situação rotineira do cotidiano real de um profissional da área, traz uma motivação para aprender e incentiva a cooperação entre os alunos [21]. Adicionalmente, os problemas devem ser relevantes com conceitos e objetivos que se relacionem com o conteúdo [23]. Para iniciar a metodologia por meio de problemas é imprescindivel identificar quais os principais conceitos que o aluno deve compreender, lidando com questões e preocupações reais.

\section{ALUNOS COM DEDICAÇÃO PARCIAL}

Alunos com dedicação parcial são caracterizados por alunos que dedicam-se parcialmente em suas atividades acadêmicas. Em linhas gerais, são alunos que dividem seu tempo entre estudo e outras ocupações que sejam consideradas para eles de suma importância. Autores como [24], [2], [25] abordam em suas obras que o trabalho dificulta a relação do aluno com o seu meio acadêmico, pois além do aluno não usufruir totalmente de sua condição de estudante, ainda tem seu aprendizado afetado.

Nesta perspectiva, pode-se afirmar que indivíduos que trabalham e estudam são alunos com dedicação parcial. Entretanto, pode-se acrescentar nesta mesma categoria donas de casa ou mães que estudam, pois apesar de não manterem um vínculo empregatício 
tendem a dedicar-se parcialmente aos estudos devido ao fluxo de ocupação diária.

A partir do momento em que o estudante tem que dividir seu tempo entre trabalho e estudo, o segundo acaba ficando parcialmente afetado. Em conformidade com [2], o trabalho dificulta o envolvimento do aluno no meio acadêmico em sua totalidade, tornando o aprendizado um tanto quanto mais complexo. Além disso, o trabalho só é desejável se relacionado ao processo educativo, senão acaba prejudicando o desempenho por não estar totalmente dentro da universidade, o que o torna um aluno com dedicação parcial.

Existem diferenças nas condições de estudo entre jovens que apenas estudam e os que estudam e mantém um vínculo empregatício. O primeiro aspecto é a diferença entre o tempo que estes jovens utilizam para dedicar-se aos estudos fora do horário de aula e âmbito escolar [24]. Para [26], mesmo que o aluno tente conciliar trabalho e estudo, o cansaço físico e mental do dia-a-dia destes acabam atrapalhando a dedicação essencial durante o período acadêmico.

Conforme [27], um dos motivos para o índice elevado de evasão é a incompatibilidade de horário entre trabalho e o curso superior. Outro fator apontado é a dificuldade financeira, o que pode ocasionar o aluno ter que se dispor a conciliar trabalho e o estudo. [28] diz que conciliar trabalho e estudo é uma das causas para que ocorra evasão. Ao conciliar trabalho e estudo o rendimento escolar pode ficar abaixo do esperado, o que pode levar à desistência do curso [27].

O estudo se torna mais complexo para o aluno que concilia trabalho e estudo, o que pode influenciar em sua formação. Desta maneira, ao ingressar no mercado de trabalho pode sentir mais dificuldade para realizar determinadas tarefas, pois há lacunas em sua formação acadêmica [2]. Neste contexto, pode ser que o egresso com dedicação parcial se considere despreparado para certas funções.

\subsection{Estudo sobre os fatores dificultadores do aprendizado para alunos com dedicação parcial}

Atualmente, espera-se que os alunos saiam com diferenciais da universidade, como ter uma boa comunicação e o senso de trabalho em equipe. Entretanto, em algumas universidades alcançar que os alunos saiam com diferenciais, já que a realidade nem sempre são de alunos que dedicam-se somente aos estudos, ao contrário observa-se um elevado índice de alunos que necessitam trabalhar e estudar para a busca de um futuro satisfatório.

Um estudo foi realizado para identificar os fatores que dificultam o aprendizado de alunos com dedicação parcial. O levantamento foi realizado com os alunos da Universidade Estadual de Mato Grosso do Sul (UEMS) - Câmpus Nova Andradina, nos cursos de Computação e Matemática realizados no período noturno, a fim de obter dados sobre estes fatores.

Um dos questionamentos realizados aos alunos foi em relação ao vínculo empregatício. Na Figura 1 são apresentados os resultados referentes a este questionamento. Pode-se observar um percentual alto de alunos que trabalham e estudam nesta universidade. Dessa maneira, pouco mais de $70 \%$ podem ser considerados alunos com dedicação parcial, enquanto pouco menos de $30 \%$ podem se dedicar apenas aos estudos. Quando são considerados apenas os dados dos alunos do curso do Computação, o percentual de alunos com dedicação parcial é maior, cerca de $85 \%$.

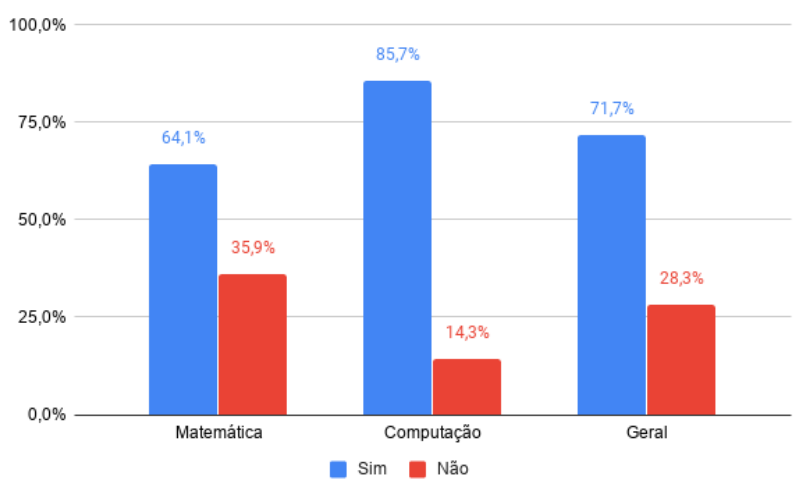

Figure 1: Quantitativo de alunos com dedicação parcial. Fonte: os autores.

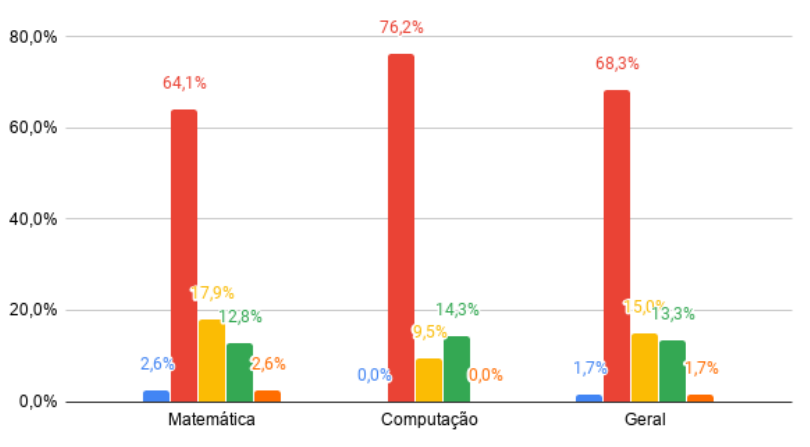

Não estudo fora do horário de aula Só estudo para provas e trabalhos Até 1 hora Entre 1 e 4 horas Acima de 4 horas

Figure 2: Quantitativo de estudo diária. Fonte: os autores.

A próxima questão é sobre a quantidade média de horas que o aluno costuma dedicar aos estudos por dia. Os resultados são ilustrados na Figura 2, em que é notado que quase 70\% dos alunos que responderam ao questionário estudam somente para realizar provas e trabalhos. É possível que este percentual esteja relacionado ao da Figura 1 sobre os alunos que trabalham, uma vez que a porcentagem dos alunos que trabalham e os que estudam apenas para provas e trabalho coincidem. Com isso, o trabalho pode ser um fator que dificulta o tempo de dedicação do aluno ao estudo.

Em seguida, foi questionado aos alunos sobre os fatores que costumam influenciar na sua aprendizagem. Na Figura 3 é exibida uma visão geral das respostas em relação ao que mais dificulta o ensino aprendizagem dos alunos. Uma observação importante é que os alunos poderiam considerar mais de uma opção. De modo geral, o fator mais apontado foi o "cansaço mental" seguidos pela "falta de tempo para o estudo" e pelo "cansaço físico". Esses fatores podem ser remetidos ao trabalho, já que a maioria deles, como trabalhos domésticos ou repetitivos podem causar este tipo de cansaço. Além 
disso, os alunos que não têm tempo para os estudos, na maioria das vezes, são alunos que mantém suas atividades diárias durante o dia e a noite estudam.

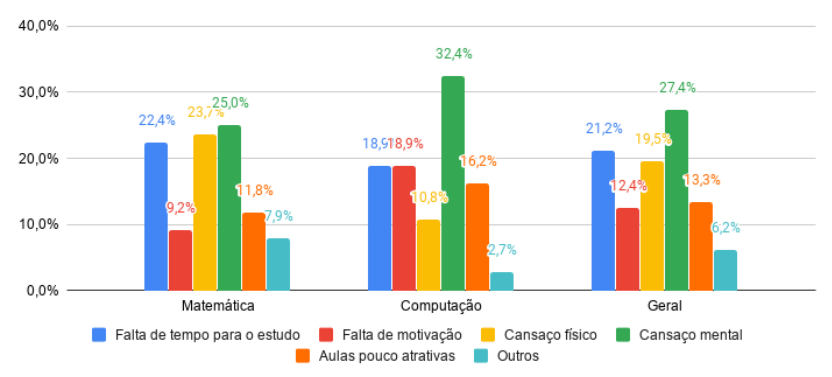

Figure 3: Fatores dificultadores da aprendizagem. Fonte: Os autores.

Para finalizar foi realizado um questionamento acerca da utilização de aplicativos para a fixação do conhecimento. Neste foi obtido um percentual elevado com respostas positivas, conforme é apresentado na Figura 4.

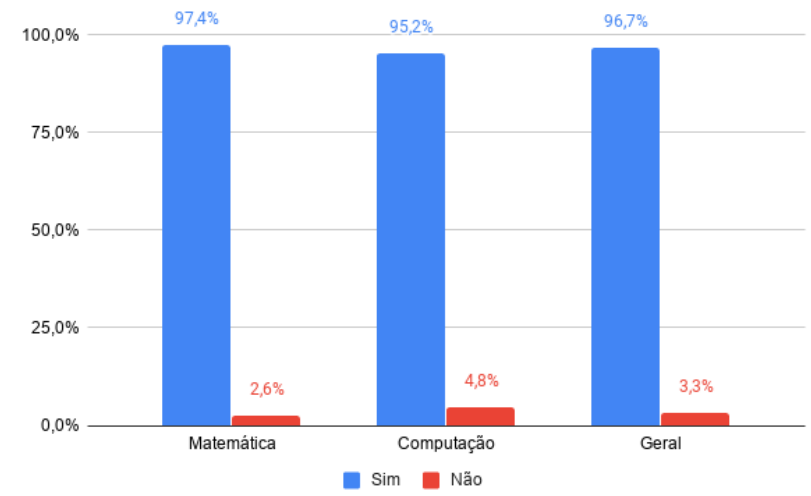

Figure 4: Quantitativo de alunos que utilizariam aplicativos para fixação de um conhecimento.

Com este estudo foi possível perceber que o trabalho é um fator complexo, que dificulta o envolvimento do aluno com o meio acadêmico ocasionando diversos outros fatores dificultadores da aprendizagem, como o cansaço mental e físico e a falta de tempo para os estudos. Além disso, a utilização de aplicativos para a fixação de um conhecimento pode ser considerado um grande aliado ao ensino, já que de acordo com o estudo foi bem aceito pelos acadêmicos.

\section{PROPOSTA: APRENDIZAGEM BASEADA EM PROBLEMAS E COLABORATIVA NO ENSINO DE ENGENHARIA DE SOFTWARE}

A proposta deste trabalho foi realizada a partir da análise dos resultados da pesquisa sobre os fatores que dificultam a aprendizagem, levando em consideração os alunos com dedicação parcial. O fluxo de execução das atividades é exposto na Figura 5, a qual foi realizada tomando como base conceitos da aprendizagem baseada em problemas e colaborativa para o conteúdo de teste de software.

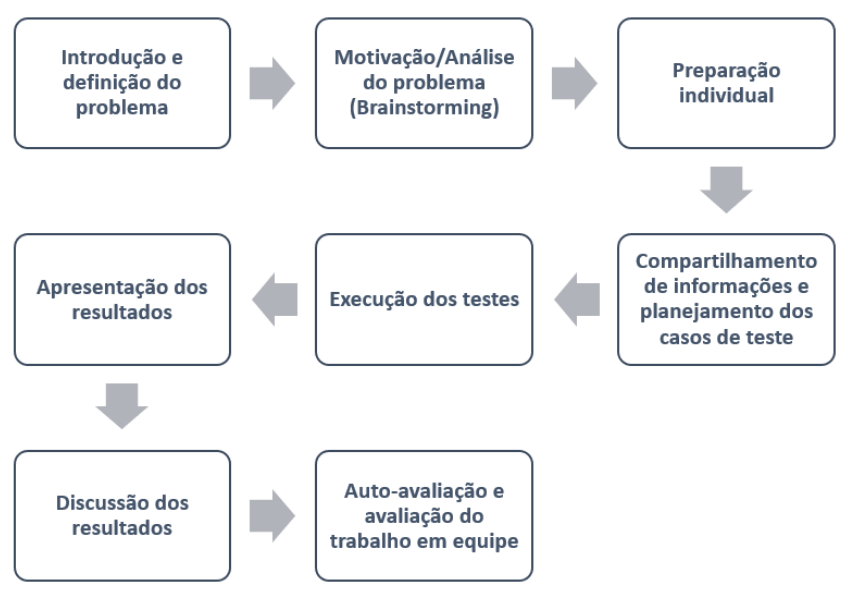

Figure 5: Procedimento para aplicação da Aprendizagem Baseada em problemas no conteúdo de teste de software. Fonte: Os autores.

O conteúdo escolhido para a aplicação da proposta foi o teste de software, pois no processo de desenvolvimento de software, os testes são classificados como uma das partes mais custosas, que mais necessitam de tempo e além disso dispõe de poucos profissionais capacitados no mercado. Assim, utilizando-se de um processo mais dinâmico de aprendizado, pode-se proporcionar aos alunos experiências que os auxiliem na fixação do conhecimento.

Antes da aplicação da proposta, o facilitador deve dividir a turma em equipes pequenas de 3 a 5 integrantes, para que haja a colaboração e todos os envolvidos possam interagir com o problema. A referida proposta foi dividida em três partes, sendo o primeira a preparação, a segunda execução e a terceira a análise dos resultados. A preparação se subdividiu na introdução e definição do problema, motivação/análise do problema por meio de um brainstorming, preparação individual e compartilhamento de informações e então o planejamento dos casos de teste em grupo. Já a execução consiste apenas na fase da execução dos testes e por fim os resultados que são constituídos das apresentações e discussões dos resultados obtidos e das avaliações.

Introdução e definição do problema: esta fase tem a função de apresentar um cenário e em seguida informar o problema, ou problemas, que serão trabalhados em aula. Além disso, deve-se explicar os referidos problemas às equipes de modo que todos os integrantes entendam o escopo do cenário e o problema.

Motivação/Análise do problema (brainstorming): nesta fase o tutor (motivador) deve exercer seu papel motivando as equipes por meio de exemplos sobre a importância de buscar o conhecimento para resolução do problema proposto. Neste momento pode ocorrer um brainstorming para as equipes exporem suas ideias sobre o problema a partir de suas experiências. Após a discussão e explanação de conhecimentos, o objetivo é que as equipes estejam instigadas pela busca do conhecimento. 
Preparação Individual: com as equipes motivadas e visando o compartilhamento de informações, é necessário realizar a preparação individual. Para que haja uma aprendizagem colaborativa todos os integrantes da equipe devem compartilhar um novo conhecimento.

Compartilhamento de informações e Planejamento dos casos de teste em grupo: nesta fase é realizado o compartilhamento das informações obtidas a partir da preparação individual. Os alunos são incentivados a dividirem pontos, aos quais eles acreditam ser importantes. Deve ser realizado o planejamento sobre qual técnica de teste deve ser utilizada para a resolução do problema e como essa técnica será aplicada para alcançar resultados esperados.

Execução dos Casos de Teste: após o planejamento devem ser aplicadas as técnicas escolhidas e realizados os casos de teste a fim de solucionar o problema proposto na primeira fase do ciclo. Esta fase deve ser realizada até que a equipe atinja seus objetivos.

Apresentação dos Resultados: nesta fase são apresentados os resultados por meio de tabelas, relatórios ou outros meios que a equipe acredite ser pertinente, para que possam ser discutidos na fase de Discussão dos Resultados. Por fim, na e tapa de autoAvaliação e avaliação do trabalho em Equipe será realizada a análise do trabalho individual e do trabalho em grupo.

Como fora apresentado no modelo acima, deve ser realizada uma preparação individual para que os alunos cheguem em aula com conhecimento necessário. Desta maneira pode haver as trocas de informações e a aplicação da aprendizagem colaborativa. Entretanto, como discutido na Seção 4.1, alunos com dedicação parcial sofrem com a falta de tempo para os estudos, e consequentemente a preparação individual fica prejudicada.

Pensando nisso, a preparação individual foi realizada com o apoio de um aplicativo desenvolvido durante a execução do trabalho. Este contém teorias condensadas sobre o conteúdo necessário para a discussão do problema. O apoio deste aplicativo foi de grande auxílio na fase de preparação individual, principalmente para alunos com tempo escasso.

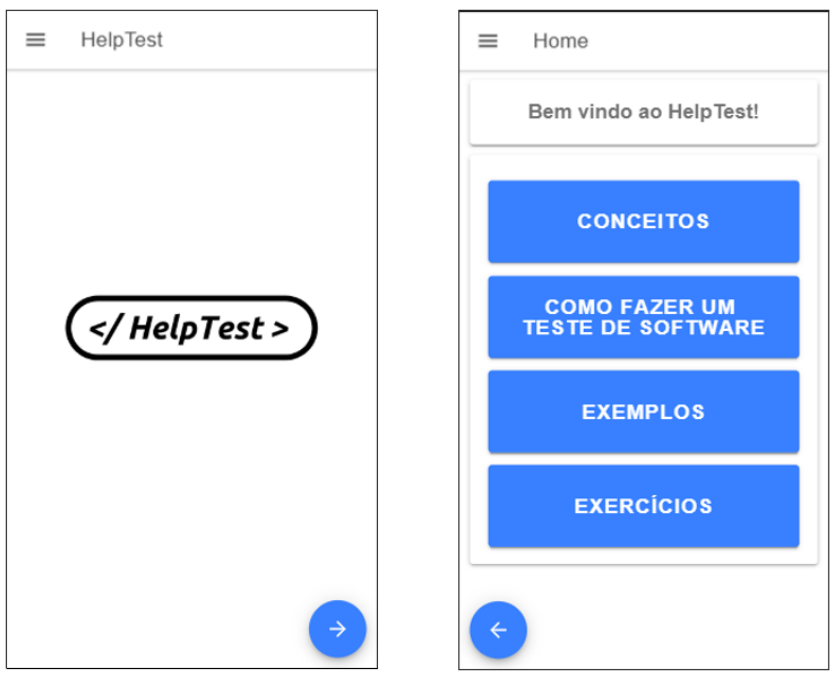

Figure 6: Interface inicial e interface principal. Fonte: Os autores.
Na Figura 6, são apresentadas as interfaces iniciais do aplicativo. Por meio destas, é possível revisar conceitos gerais sobre teste de software e de elaboração de casos de teste. Além disso, são apresentados exemplos práticos e exercícios sobre a teoria.

\section{DISCUSSÕES E RESULTADOS}

A aplicação da proposta foi realizada juntamente com o ensino de conceitos de testes de software. Para realizar a aplicação, os alunos foram divididos em duas turmas, que realizaram atividades práticas de teste de software unitário. Na turma 1 não foi utilizado o auxílio do aplicativo, eles contaram apenas com o conhecimentos anteriores. Além disso, não foi aplicado o conceito de aprendizagem colaborativa, a fim de realizar comparações entre as turmas.

\subsection{Atividades realizadas com a turma 1}

Inicialmente, foi apresentada a ferramenta $\mathcal{f U n i t} 5^{1}$ - um framework aplicado no desenvolvimento de casos de teste. Em seguida, foi disponibilizado uma lista de dez exercícios. O intuito da aplicação desses exercícios não era a codificação, mas sim o projeto de casos de teste de software.

Os exercícios propostos, foram divididos em grupos, no qual a finalidade do primeiro grupo era somente na familiarização dos alunos com a ferramenta e na estruturação dos casos de teste de software. Em consequência disto, esses exercícios já continham alguns testes codificados e foi solicitado apenas para que os alunos acrescentassem casos de teste.

Para os exercícios do próximo grupo, foi pedido para que os alunos projetassem todos os casos de teste, o que acarretou em algumas dúvidas e dificuldades que foram sendo sanadas ao longo da aplicação. Os exercícios do grupo final consistiram na realização da manutenção de alguns dos códigos realizados. Houve uma mudança no enunciado do exercício e os alunos precisaram analisar o impacto nos casos de teste projetados. Essa atividade simulava uma mudança na regra de negócio.

Ao longo da aplicação, metade dos alunos conseguiram identificar defeitos inseridos no código. Isso foi um ponto positivo, pois os alunos ao receberem e interpretarem os problemas, além de identificar os defeitos ainda conseguiram efetuar a correção. Isso traz uma contribuição para sua formação, adquirindo habilidades que o mercado procura.

\subsection{Atividades realizadas com a turma 2}

Na turma 2 foi utilizado o método apresentado na Sessão 5. Foi realizada a introdução dos problemas propostos, seguido de um brainstorming, além da preparação individual que foi realizado com o auxílio do aplicativo HelpTest. Logo após, foi realizada a etapa de projeto e execução dos testes. O método foi finalizado com a aplicação de um questionário para a obtenção de resultados.

A turma 2 também utilizou o mesmo conjunto de exercícios que foi realizado pela turma 1. Entretanto, a segunda turma utilizou o auxílio do aplicativo HelpTest, que foi disponibilizado 3 dias antes para que os alunos pudessem utilizá-lo. No dia da aplicação, inicialmente foi entregue um questionário a respeito do aplicativo.

Posteriormente, uma breve introdução a conceitos pertinentes da pesquisa foi realizada, sendo eles: o que são alunos com dedicação

\footnotetext{
${ }^{1}$ https://junit.org/junit5/
} 
parcial?, metodologias ativas, aprendizagem baseada em problemas e aprendizagem colaborativa. Estes conceitos foram apresentados para que os alunos, posteriormente, fossem capazes de responder aos questionamentos aplicados ao términos das atividades.

Em seguida uma breve introdução sobre a ferramenta $\mathcal{f}$ Unit5 foi realizada, visando a resolução dos exercícios. Apesar dos exercícios serem os mesmos que foram aplicados para a turma 1, dessa vez os alunos precisaram discutir sobre a técnica de teste que seria aplicada, levando em consideração todos os conhecimentos adquiridos. Após as discussões os alunos resolveram aplicar a técnica funcional juntamente com o teste de unidade.

Logo após, os alunos começaram a projetar os casos de teste assim como a turma 1 , já que os exercícios propostos eram os mesmos. Assim como a turma 1, a turma 2 também apresentou algumas dificuldades tanto na utilização da ferramenta quanto na elaboração dos casos de testes. Entretanto as dificuldades foram trabalhadas colaborativamente entre os alunos.

Após o término da aplicação, os alunos responderam ao questionário referente à proposta. Um dos questionamentos foi se o acadêmico se considera um aluno com dedicação parcial, visto que muitos desses não tinham conhecimento até o momento em que foi apresentado tal conceito. Logo, $100 \%$ dos alunos responderam que se consideravam alunos com dedicação parcial.

Já uma outra questão era relacionada novamente ao aplicativo. Esta era uma questão aberta, na qual os alunos poderiam expressar suas opiniões em relação aos benefícios que o aplicativo lhes trouxera. Nesta obteve-se feedback positivo, já que a Aluno A relatou que um dos benefícios foi o "estudo em qualquer lugar e a praticidade". O Aluno $B$, afirmou que um de seus benefícios é "poder utilizar no trabalho, por não ser algo que demande muito tempo, nos tempos vagos além de poder utilizar a caminho da faculdade ou serviço". Já o Aluno $C$ elogia "a facilidade para fixação, rapidez no uso e a apresentação dos principais conceitos, são ótimos para entender de forma rápida".

As Figuras 7 e 8 ilustram as opiniões dos alunos sobre as metodologias apresentadas em aula, foi questionado se as mesmas proporcionaram mais experiências ao conteúdo abordado. Para a aprendizagem colaborativa $75 \%$ das respostas foram que os alunos estavam satisfeitos, enquanto apenas $25 \%$ responderam que não obteve nenhuma experiência. Já em relação a aprendizagem baseada em problemas, $75 \%$ das respostas foram que foi satisfatória as experiências obtidas a partir dessa metodologia enquanto $25 \%$ dos alunos responderam que foi parcialmente.

\subsection{Considerações finais}

A Tabela 1 apresenta a quantidade total de casos de testes elaborados por aluno. Os alunos da turma 1 realizaram mais casos de teste em relação a turma 2. Entretanto, como o tempo de aplicação foi diferente para as duas turmas (devido a problemas de disponibilidade dos participantes), foi calculada a taxa de realização de casos de teste.

A fórmula para o cálculo foi a quantidade de casos teste realizados / tempo (em minutos). Desta maneira, foi possível analisar a quantidade de casos testes realizados por minuto. $\mathrm{O}$ total de casos de teste realizados pela turma 1 foi de 163 casos de teste
Na sua opinião, você considera que a metodologia ativa: aprendizagem colaborativa trouxe mais experiências sobre o conteúdo abordado? 4 respostas

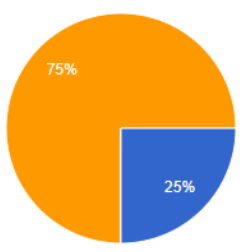

- Nenhum

Parcialme

Satisfeito

- Totalmente Satisfeito
Figure 7: Nível de satisfação acerca da aprendizagem colaborativa em relação a of erecer mais experiências sobre o conteúdo abordado. Fonte: Os autores.

Na sua opinião, você considera que a metodologia ativa: aprendizagem baseada em problemas trouxe mais experiências sobre o conteúdo abordado?

4 respostas
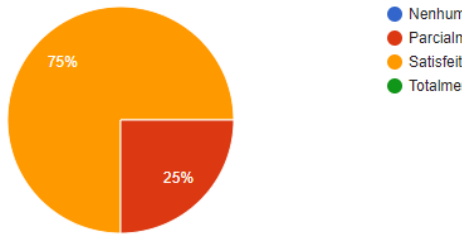

- Parcialmen

- Totalmente Satisfeito

Figure 8: Nível de satisfação acerca da aprendizagem baseada em problemas em relação a oferecer mais experiências sobre o conteúdo abordado. Fonte: Os autores.

realizados em 170 minutos, enquanto a turma 2 realizou 105 casos de teste em 90 minutos. A Tabela 1 apresenta estes resultados.

\begin{tabular}{|c|c|c|c|c|c|c|c|c|}
\hline & \multicolumn{3}{|c|}{ Turma 1 } & \multicolumn{5}{c|}{ Turma 2 } \\
\hline Aluno & $\mathbf{1}$ & $\mathbf{2}$ & $\mathbf{3}$ & $\mathbf{4}$ & $\mathbf{5}$ & $\mathbf{6}$ & $\mathbf{7}$ & $\mathbf{8}$ \\
\hline Qtde_Casos_Teste & 21 & 70 & 27 & 45 & 30 & 34 & 20 & 21 \\
\hline $\begin{array}{c}\text { Total de casos de teste } \\
\text { realizados por aluno } \\
\text { (por minuto) }\end{array}$ & 0,12 & 0,41 & 0,15 & 0,26 & 0,33 & 0,37 & 0,22 & 0,23 \\
\hline $\begin{array}{c}\text { Total de casos de teste } \\
\text { realizados por turma } \\
\text { (por minuto) }\end{array}$ & \multicolumn{3}{|c|}{$\mathbf{0 , 9 5}$} & \multicolumn{5}{c|}{$\mathbf{1 , 1 6}$} \\
\hline
\end{tabular}

Table 1: Relação da quantidade de casos teste desenvolvidos por aluno e por turma. Fonte: Os autores.

Conforme observado na Tabela 1 a turma 2 obteve resultados mais lineares em relação a turma 1 . O desempenho da turma 2 também pode ser considerado mais satisfatório, se considerada a taxa de realização de casos de teste.

\section{CONCLUSÃO}

Alunos que não podem se dedicar totalmente ao estudo acabam tendo o seu desempenho parcialmente afetado. Isso pode acarretar 
em algumas desvantagens em suas carreiras futuras. Todavia, a universidade deve se empenhar para que todos os alunos, sejam esses com dedicação parcial ou não, saiam com os conhecimentos equivalentes. A partir disso, foi realizada uma proposta que envolvesse os alunos no centro do aprendizado, com a aplicação de metodologias ativas.

Neste trabalho foi aplicada a aprendizagem colaborativa, desta forma os alunos puderam aprender a partir da troca de experiências. Além disso, foi possível desenvolver habilidades na comunicação que é um dos pontos chaves para o trabalho em equipe. Também foi utilizada a aprendizagem baseada em problemas, em que os resultados obtidos foram satisfatórios. A aplicação apresentou uma boa dinâmica, já que trabalha com o problema precedendo a explicação, permitindo que os alunos busquem uma solução, lidando com eventuais problemas.

$\mathrm{Na}$ aprendizagem baseada em problemas é comum professores ou tutores fornecerem determinados conteúdos precedendo a aula. Durante o horário de aula, os alunos são capazes de discutir sobre o conhecimento adquirido ou tirar dúvidas. Por esse motivo, e pensando na realidade de alunos com dedicação parcial, que de acordo com a pesquisa realizada não dispõem de muito tempo para os estudos, foi criado um aplicativo com conteúdo sucinto e de fácil entendimento. Por meio da análise dos resultados obtidos, foi possível constatar que o aplicativo empregado em conjunto com metodologias ativas trouxe grandes vantagens por trazer praticidade, podendo ser usado nos tempos vagos.

Utilizar aplicativos com propósito de apoiar a fixação de conhecimento pode ser um recurso tanto para docentes quanto para discentes. Para os docentes é um meio alternativo de transmitir conhecimento, para o discente é uma maneira mais rápida e prática de acessar a informação no momento que achar necessário. Em relação às metodologias ativas, a aprendizagem baseada em problemas trouxe resultados mais satisfatórios em relação a aprendizagem colaborativa. Visto que, de acordo com os resultados obtidos os alunos compreenderam e exploraram melhor os recursos desta metodologia.

Em comparação às aulas com e sem o apoio das metodologias ativas, tivemos resultados satisfatórios em relação a proposta apresentada neste artigo. A partir da análise realizada na turma 2 (a qual foi feita a aplicação da proposta), é observada uma taxa maior de realização de casos de teste por minuto em relação a turma 1. Adicionalmente, a turma 2 foi apresentada a novos conceitos de metodologias ativas.

\section{REFERENCES}

[1] R.R Chelotti, Alcântara E.G, and R. A Coutinho. Aprender a apren-der:estudos sobre aprendizagem. MS: Ed. UFMS, 2006.

[2] Helena Sampaio and Ruth C.L. Cardoso. Estudantes universitários e o trabalho. Disponível em: http://www.anpocs.org.br/portal/publicacoes/rbcs_00_26/rbcs26 3.htm. Acesso em: 25 de maio de 2018, 2011.

[3] Neusi Aparecida Navas Berbel. As metodologias ativas e a promoção da autonomia de estudantes. Semina: Ciências Sociais e Humanas, 32:25-40, 11 2011. doi: 10.5433/1679-0359.2011v32n1p25.

[4] José Morán. Mudando a educação com metodologias ativas. In Souza, Carlos Alberto de; Morales, Ofélia Elisa Torres. Convergências Midiáticas, Educação e Cidadania: aproximações jovens., pages 15-33, 2015

[5] Patricia Torres, Paulo Alcantara, and Esrom Adriano Freitas Irala. Grupos de consenso: Uma proposta de aprendizagem colaborativa para o processo de ensinoaprendizagem. Revista Diálogo Educacional, 4:129, 07 2017. doi: 10.7213/rde.v4i13. 7052.
[6] Maria Elizabeth Bianconcini de Almeida and Maria Elisabette Brito Prado. Criando situações de aprendizagem colaborativa. In Workshop em Informática na Educação - WIE - 2003, pages 53-60, 2003. doi: 10.5753/cbie.wie.2003.53-60.

[7] E. Mazur. Peer Instruction: A Revolução da Aprendizagem Ativa. Penso Editora, 2015. ISBN 9788584290635. URL https://books.google.com.br/books?id= K3GFCgAAQBAJ.

[8] Roberto Paulo Alcântara, Maria Marques Lilia Siqueira, and Suzana Valaski. Vivenciando a aprendizagem colaborativa em sala de aula:experiÊncias no ensino superior. Revista Diálogo Educacional, 4:169, 07 2017. doi: 10.7213/rde.v4i12.6986.

[9] Raquel Freitas. Ensino por problemas: uma abordagem para o desenvolvimento do aluno. Educação e Pesquisa, 38(2):403-418, jun. 2012. doi: 10.1590/ S1517-97022011005000011. URL http://www.revistas.usp.br/ep/article/ view/47885.

[10] Luis Ribeiro and Camargo Ribeiro. Aprendizagem baseada em problemas (pbl) na educaÇÃo em engenharia. Revista de Ensino de Engenharia, 27:23-32, 2008.

[11] Neusi Aparecida Navas Berbel. A problematização e a aprendizagem baseada em problemas: Diferentes termos ou diferentes caminhos? Interface - Comunicação, Saúde, Educação, 2, 02 1998. doi: 10.1590/S1414-32831998000100008.

[12] Edmundo Escrivão Filho and Luis Roberto De Camargo Ribeiro. Aprendendo com pbl-aprendizagem baseada em problemas: Relato de um experiência em cursos de engenharia da eesc-usp. pages 23-30, 2016.

[13] J.E.D. Bordenave and A.M. Pereira. Estratégias de ensino-aprendizagem. Vozes, 1998. ISBN 9788532601544. URL https://books.google.com.br/books?id= JFBQSwAACAAJ.

[14] Terezinha Richartz. Metodologia ativa: a importância da pesquisa na formação de professores. Revista da universidade vale do rio verde, 01 2015. doi: 10.5892/ ruvrd.v13i1.2422.

[15] Ana Laura Schliemann and Jorge Luiz Antonio. Metodologias ativas na Uniso : formando cidadãos participativos. EDUNISO: Editora da Universidade de Sorocaba, 2016. ISBN 978-85-61289-29-4. URL https://docplayer.com.br/54264566Metodologias-ativas-na-uniso-formando-cidadaos-participativos.html.

[16] A. F. Zorzo, D. Nunes, E. Matos, I. Steinmacher, J. Leite, R. M. Araujo, R. Correia, and S. Martins. Referenciais de Formação para os Cursos de Graduação em Computação. Sociedade Brasileira de Computação (SBC), 2017. ISBN 9788576694243.

[17] Ian Sommerville. Engenharia de Software. 9. ed. São Paulo: Pearson Prentice Hall, 2011.

[18] Severino Paiva and Álvaros Medeiros. Escollab: Uma metodologia colaborativa voltada para o ensino de engenharia de software. In Simpósio Brasileiro de Informática na Educação - SBIE, pages 630-639, Nov 2011. doi: 3/cbie.sbie.2011.25p.

[19] Simone de França Tonhão. Uma abordagem híbrida para o ensino de engenharia de software, 2018. Monografia (Licenciado em Computação), UEMS (Universidade Estadual de Mato Grosso do Sul), Nova Andradina, Brasil.

[20] Hong Huang and Dongyong Yang. Teaching design patterns: A modified PBL approach. In Proceedings of the 9th International Conference for Young Computer Scientists, ICYCS 2008, Zhang Fia Jie, Hunan, China, November 18-21, 2008. IEEE Computer Society, 2008. ISBN 978-0-7695-3398-8. URL http://ieeexplore.ieee.org/

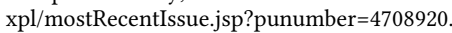

[21] Yikui Zhang and Yiqin Liu. Management enhanced double pbl based reform in advanced programming design course. In Proceedings of the 2012 IEEE 14th International Conference on High Performance Computing and Communication \& 2012 IEEE 9th International Conference on Embedded Software and Systems, HPCC 12, pages 1658-1663. IEEE Computer Society, 2012. ISBN 978-0-7695-4749-7.

[22] Simone C. dos Santos, Ana Cláudia Monte, and Ariane Nunes Rodrigues. A PBL approach to process management applied to software engineering education. In IEEE Frontiers in Education Conference, FIE 2013, Oklahoma City, Oklahoma, USA, October 23-26, 2013, pages 741-747, 2013.

[23] S. C. dos Santos, F. Furtado, and W. Lins. xpbl: A methodology for managing pbl when teaching computing. In 2014 IEEE Frontiers in Education Conference (FIE), pages 1-8, Los Alamitos, CA, USA, oct 2014. IEEE Computer Society. doi: 10.1109/FIE.2014.7044178. URL https://doi.ieeecomputersociety.org/10.1109/FIE. 2014.7044178 .

24] Ricardo Luis de Azevedo da Rocha, Fernanda Oliveira Simon, and Dirceu da Silva. Alunos que trabalham $\mathrm{x}$ alunos que só estudam: como eles avaliam os cursos de engenharia? 2004.

[25] Sabrina Favéro de Freitas. Universitários ingressantes trabalhadores e não trabalhadores: um grupo homogêneo ou heterogêneo?, 2014. Monografia (Licenciado em Pedagogia), Universidade estadual de campinas faculdade de educação, Campinas, Brasil.

[26] Nyedja Nara Furtado de Abrantes. Trabalho e estudo: Uma conciliaÇÃo desafiante. Disponível em: http://www.editorarealize.com.br/revistas/fiped/trabalhos/ ed3d2c21991e3bef5e069713af9fa6ca.pdf. Acesso em: 25 de maio de 2018, 2012.

[27] Fernando Coutinho Garcia and Elbe Figueiredo Brandão Santiago. Mecanismo de enfrentamento a evasão no ensino superior público: Inserção do conteúdo sobre profissões no ensino médio. Simpósio de Excelência em Gestão e Tecnologia, 102015.

[28] Augusto Guimarães. Quais as causas da evasão no ensino superior? Disponível em: https://crmeducacional.com/2018/11/19/evasao/. Acesso em: 12 de junho de 2018, 2018. 